\title{
Gauss' Theorem in a General Space-time
}

\author{
By H. S. Ruse, University of Edinburgh. \\ (Received 27th February, 1935. Read 1st March, 1935.)
}

\section{§1. Introduction.}

In classical mechanics Gauss' Theorem for a gravitational field states that, if $S$ is a closed surface and $N$ the component of gravitational force along the outward normal, then

$$
\int_{S} N d S=-4 \pi \beta M
$$

where $\beta$ is the Newtonian constant of gravitation and $M$ is the total mass inside $S$. This result has recently been extended to general relativity by E. T. Whittaker, ${ }^{1}$ who, however, considered only the case of a statical gravitational field, the line-element of which is given $b^{2}$

$$
d s^{2}=U d t^{2}-a_{\mu \nu} d x^{\mu} d x^{\nu}, \quad(\mu, \nu=1,2,3)
$$

where the coefficients $U$ and $a_{\mu \nu}$ are independent of $t$. It is not immediately clear from his work whether the results are extensible to more general space-times. It is the purpose of this paper to elucidate this point, the problem being to find a formula for a general space-time

$$
d s^{2}=g_{i j} d x^{i} d x^{j} \quad(i, j=0,1,2,3)
$$

which reduces to that obtained by Whittaker when (1.3) is of the form (1.2). In doing so it is difficult to avoid an appearance of artificiality, which is mainly due to the fact that the theorem involves a definition of gravitational force, not relative to a single observer, but to an infinite set whose world-lines are not geodesics; these world-lines form, in fact, a congruence of curves in space-time which is largely arbitrary. No claim is therefore made that the general theorem of this paper is as interesting from the physical point of view as the special case of it considered by Whittaker.

1 Proc. Roy. Soc. (A) 149 (193̄̃), 384.

2 The summation convention for repeated suffixes is employed throughout this paper, and the velocity of light in vacuo is taken as unity. Greek suffixes will take the values 1 to 3 only. Latin suffixes, used below, take the values 0 to 3 . 
Indeed, it is described as an extension of Gauss' Theorem to general relativity only because of its close formal resemblance to the classical result; from the physical point of view it may not be the most suitable generalisation of Gauss' Theorem. ${ }^{1}$ Nevertheless, it is hoped that the following work may be of some interest in itself, since it expresses Whittaker's result in proper tensor form and throws light on the geometrical basis of his theory. It will be seen that the generalised Gauss' Theorem is a not uninteresting special case of Green's Theorem in four dimensions.

\section{§2. The fundamental observers: gravitational force.}

As remarked by Whittaker, the gravitational force experienced by any observer depends upon his velocity and acceleration as well as upon his position. So in forming an integral of gravitational force over a surface $S$ it is necessary to specify at every point an observer relative to whom the force is measured. In the statical world (1.2) Whittaker defines these fundamental observers to be "at rest," that is, to have world-lines $x^{1}=$ const., $x^{2}=$ const., $x^{3}=$ const. Relative to them the four-dimensional world is partitioned into space and time. The latter is measured by $t$, and, at any instant, "space" is the hypersurface $t=$ const. Now it is to be noticed that the world-lines $x^{\mu}=$ const. of the observers are the orthogonal trajectories of the spatial sections $t=$ const. of the statical world. In generalising to any space-time

$$
d s^{2}=g_{i j} d x^{i} d x^{j}
$$

we therefore choose as fundamental observers those whose worldlines are the orthogonal trajectories of a singly-infinite family of hypersurfaces

$$
f\left(x^{0}, x^{1}, x^{2}, x^{3}\right)=\text { const., }
$$

which are then, relative to the observers, the spatial sections of the four-dimensional world.

Let $\lambda^{i}$ be the unit four-vector in the direction of the world-line of the fundamental observer at any point $\left(x^{i}\right)$. Since $\lambda^{i}$ is unit and necessarily time-like, we have

$$
g_{i j} \lambda^{i} \lambda^{j}=1
$$

1 I am indebted to a referee whose criticism of the physical aspect of this paper led to the re-writing of the latter half of $\S 1$. 
and since $\lambda^{i}$ is normal to that hypersurface $f=$ const. which passes through $\left(x^{i}\right)$, we also have

where $\lambda_{i}=g_{i j} \lambda^{j}$ and

$$
\lambda_{i}=U^{\frac{3}{3}} \frac{\partial f}{\partial x^{i}},
$$

$$
U \equiv\left(\Delta_{1} f\right)^{-1} \equiv\left(g^{i j} \frac{\partial f}{\partial x^{i}} \frac{\partial f}{\partial x^{j}}\right)^{-1}
$$

$U$ is thus the reciprocal of Beltrami's first differential parameter of $f$. The factor $U^{\frac{1}{3}}$ is inserted in (2.4) in order that $\lambda_{i}$ should satisfy (2.3), and the notation $U$ is chosen because, as is shown in $\S 5, \Delta_{1} f$ is precisely the reciprocal of the coefficient $U$ of $d t^{2}$ when the metric is of the form (1.2).

The differential equations of the world-lines of the fundamental observers are

$$
\frac{d x^{i}}{d \omega}=\lambda^{i}
$$

$d \varpi$ being the element of proper-time. The gravitational force at any world-point $\left(x^{i}\right)$, relative to the fundamental observer whose worldline passes through that point, is given by the four-vector ${ }^{1}$

$$
g^{i}=-\left[\frac{d^{2} x^{i}}{d \varpi^{2}}+\left\{\begin{array}{c}
i \\
j k
\end{array}\right\} \frac{d x^{j}}{d \varpi} \frac{d x^{k}}{d \varpi}\right]
$$

Using (2.6), we at once get

$$
g^{i}=-\lambda^{j}\left(\lambda^{i}\right)_{j}
$$

where $\left(\lambda^{i}\right)_{j}$ is the covariant derivative of $\lambda^{i}$. Now equation (2.3) may be written $\lambda^{i} \lambda_{i}=1$, and this, differentiated covariantly, gives $\left(\lambda^{i}\right)_{j} \lambda_{i}=0$. From (2.8) it follows that

$$
g^{i} \lambda_{i}=0 .
$$

So the four-vector $g^{i}$ representing the gravitational force is perpendicular to the world-line of the observer, and is therefore tangent to the hypersurface $f=$ const. That is, $g^{i}$ is a vector in "space," and in appropriate coordinates could be represented as a spatial threevector.

' Whittaker, loc. cit., equation (2.2). $C f$. Walker, page 173 of the present volume of these Proceedings. 


\section{§3. The surface of integration.}

In the case of the statical space-time (1.2) the surface $S$ of integration at any instant $t_{0}$ is a fixed surface lying in the instantaneous space $t=t_{0}$. Four dimensionally' it is the intersection of a tubular hypersurface generated by $\infty^{2}$ of the world-lines $x^{\mu}=$ const. $(\mu=1,2,3)$, and the hypersurface $t=t_{0}$.

In generalising to the space-time $d s^{2}=g_{i j} d x^{i} d x^{j}$ we therefore define the surface $S$ of integration to be the intersection of a spatial hypersurface $f=$ const. with a hypersurface

$$
\phi\left(x^{0}, x^{1}, x^{2}, x^{3}\right)=0
$$

which contains $\infty^{2}$ of the fundamental world-lines. Since $S$ is to be a closed surface, $\phi=0$ is a hypercylinder with lines of the congruence $\lambda^{i}$ as (curvilinear) generators, cutting all the hypersurfaces $f=$ const. orthogonally. The covariant direction-ratios of the normals to $f=$ const. and $\phi=0$ are respectively $\lambda_{i}$ and $\partial \phi / \partial x^{i}$. Since these directions are perpendicular, we have

$$
\lambda^{i} \frac{\partial \phi}{\partial x^{i}}=\mathbf{0}
$$

Let $n^{i}$ be the unit outward four-vector normal at $\left(x^{i}\right)$ to the hypersurface $\phi=0$ and therefore tangent to the hypersurface $f=$ const. passing through $\left(x^{i}\right)$. Then $n^{i}$ is a unit space-like vector, so

$$
g_{i j} n^{i} n^{j}=-1 \text {. }
$$

Also, since it is normal to $\phi=0$,

$$
n_{i}=\frac{\partial \phi}{\partial x^{i}} /\left(-\Delta_{1} \phi\right)^{\frac{1}{2}}
$$

the negative of the Beltrami differential parameter appearing in the denominator on account of the minus sign on the right-hand side of (3.2). Hence by (3.1),

$$
\lambda^{i} n_{\iota}=0
$$

1 A mental picture may be formed by imagining the number of dimensions of spacetime reduced to three. The spatial hypersurfaces $f=$ const. are then represented by a family of surfaces $f=$ const., the observers' world-lines by the orthogonal trajectories of the family, and the surface $S$ at every instant by a closed curve in one of the surfaces $f$. The orthogonal trajectories passing through this curve generate a surface which is the three-dimensional analogue of the tubular hypersurface. 
The $n^{i}$ are the components in four dimensions of the normal to the surface $S$ in the 3 -space $f=$ const. The component of gravitational force along this normal is ${ }^{1}-n_{i} g^{i}$, so it may be expected that, in the generalisation of Gauss' Theorem, the surface-integral will be

$$
\int_{S}\left(-n_{i} g^{i}\right) d V_{2}
$$

where $d V_{2}$ is the element of area of $S$. Actually it will appear that this is not the proper generalisation of Gauss' integral.

\section{§4. Gauss' Integral.}

$g^{i}$ is a vector-field defined at all points of space-time under consideration. Consider any closed hypersurface $\Omega$, and let $v^{i}$ be the unit outward normal vector defined at all points of $\Omega$. Let $d V_{3}$ be the element of surface-volume of $\Omega$, and let $d V_{4}$ be the element of volume of space-time, so that

$$
d V_{4}=(-g)^{\frac{1}{2}} d x^{0} d x^{1} d x^{2} d x^{3} .
$$

Let $Q$ be the 4-volume enclosed by $\Omega$. Then by Green's Theorem in four dimensions

$$
\int_{\Omega} \epsilon \nu_{i} g^{i} d V_{3}=\int_{Q}\left(g^{i}\right)_{i} d V_{4}
$$

where $\epsilon$ is the indicator ${ }^{2}$ of the vector $\nu^{i}$, that is, a coefficient which is equal to +1 when $\nu^{i}$ is time-like and to -1 when $\nu^{i}$ is space-like. The integrand $\left(g^{i}\right)_{i}$ on the right-hand side is the contracted covariant derivative of $g^{i}$.

Take the hypersurface $\Omega$ to be that bounded by the hypersurfaces

$$
\begin{aligned}
& f=t, \\
& \phi=0, \\
& f=t+d t,
\end{aligned}
$$

where $t, d t$ are constants and $d t$ is small. $\Omega$ then consists of three

1 The negative sign is taken because the vectors $n^{i}$ and $g^{i}$ are both space-like, and the fundamental quadratic form should thus be taken negatively $\left(d s^{2}=-g_{i j} d x^{i} d x^{j}\right)$. That this is so is most easily seen by considering the angle $\theta(=0)$ which a unit spacelike vector such as $n^{i}$ makes with itself. Since $n_{i} n^{i}=-1$, we have $\cos \theta=-n_{i} n^{i}$.

${ }^{2}$ Synge, Trans. Roy. Soc. Canada, 28 (1934), 169 ; see also p. 134. Synge takes the metric with signature $(-+++)$ instead of $(+---)$, so that with him time-like and space-like directions have respectively indicators -1 and +1 . He is apparently the first to have noticed the need for inserting the indicator $\epsilon$ in one of the integrals of Green's Theorem. 
parts, $R, \Sigma$ and $R^{\prime} ; R$ is that part of $f=t$ interior to the surface $S$ which is the intersection of $f=t$ and $\phi=0$, and is therefore the spatial 3-volume enclosed by $S ; \Sigma$ is a narrow segment of the tubular hypersurface $\phi=0$, and $R^{\prime}$ is the 3 -volume enclosed by the surface $S^{\prime}$ in which $\phi=0$ cuts $f=t+d t$.

Now over $R$ the normal $\nu^{i}$ is $\nu^{i}= \pm \lambda^{i}$, since $\lambda^{i}$ is the directionvector of the orthogonal trajectories of the hypersurfaces $f=$ const., and $R$ is a part of one of these. Similarly over $R^{\prime}$ the normal is $\nu^{i}=\mp \lambda^{i}$. Hence since $\lambda_{i} g^{i}=0$ by (2.9), the part of the left-hand side of (4.2) due to integrating over $R$ and $R^{\prime}$ is zero, and we are left with

$$
\int_{\Sigma} \epsilon \nu_{i} g^{i} d V_{3} .
$$

Now over $\Sigma$, which is a part of the hypersurface $\phi=0, \nu^{i}$ is the normal $n^{i}$ defined by (3.3). Also $\epsilon=-1$ because $n^{i}$ is space-like, so (4.2) becomes

$$
\int_{\Sigma}\left(-n_{i} g^{i}\right) d V_{3}=\int_{Q}\left(g^{i}\right)_{i} d V_{4} .
$$

Let now $\left(x^{i}\right)$ be a point on $R$, so that

$$
f\left(x^{i}\right)=t .
$$

Further, let $d \varpi$ be the normal distance from this point to $R^{\prime}$. Then $d \varpi$ has the direction $\lambda^{i}$ of the normal to $R$, so the world-point $\left(x^{i}+\lambda^{i} d \Phi\right)$ lies on $R^{\prime}$, that is, on the hypersurface $f=t+d t$. Hence

whence, using (4.4),

$$
f\left(x^{i}+\lambda^{i} d \varpi\right)=t+d t,
$$

$$
\lambda^{i} \frac{\partial f\left(x^{j}\right)}{\partial x^{i}} d \varpi=d t .
$$

Now by (2.4) and (2.5),

so by (4.5),

$$
\begin{aligned}
\lambda i \frac{\partial f}{\partial x^{i}} & =U^{\frac{1}{2}} \cdot g^{i j} \frac{\partial f}{\partial x^{i}} \frac{\partial f}{\partial x^{j}} \\
& =U^{-\frac{1}{2}}
\end{aligned}
$$

$$
d \sigma=U^{\frac{1}{2}} d t .
$$

In (4.3), $d V_{3}$ is the element of surface-volume of the segment $\Sigma$ of the hypersurface $\phi=0$, hence

$$
d V_{3}=d \varpi d V_{2}
$$

where $d V_{2}$ is the element of area of the surface $S$, that is, of the intersection of $\Sigma$ with the hypersurface $f=t$. Similarly

$$
d V_{4}=d \varpi d V_{3}
$$


where $d V_{3}$ is now the element of the spatial 3-volume $R$ enclosed by $S$. Substituting from (4.7) and (4.8) in (4.3), using (4.6) and dividing by the constant $d t$, we get

or by $(2.5)$,

$$
\int_{S}\left(-n_{i} g^{i}\right) U^{\ddagger} d V_{2}=\int_{R}\left(g^{i}\right)_{i} U^{\natural} d V_{3},
$$

$$
\int_{S}\left(-n_{i} g^{i}\right) \frac{d V_{2}}{\left(\Delta_{1} f\right)^{\frac{3}{2}}}=\int_{R}\left(g^{i}\right)_{i} \frac{d V_{3}}{\left(\Delta_{1} f\right)^{\frac{1}{2}}} .
$$

The left-hand integral may be compared with (3.5). It will be seen that the appropriate generalisation of Gauss' integral is not simply the surface-integral of the normal component of gravitational force ${ }^{1}$. But if we write

(4.10) becomes

$$
\left.\begin{array}{l}
d W_{2} \equiv d V_{2} /\left(\Delta_{1} f\right)^{\frac{1}{2}} \\
d W_{3} \equiv d V_{3} /\left(\Delta_{1} f\right)^{\frac{1}{4}},
\end{array}\right\}
$$

$$
\int_{S}\left(-n_{i} g^{i}\right) d W_{2}=\int_{R}\left(g^{i}\right)_{i} d W_{3}
$$

$d W_{2}$ and $d W_{3}$ may be called respectively the relative surface-element and volume-element, and space may be thought of as contracted (if $\Delta_{1} f>1$ ) or expanded (if $\Delta_{1} f<1$ ) relative to the fundamental observers. The left-hand side of (4.12) may then be called the relative surface-integral of the normal component of gravitational force.

\section{§5. Gauss' Theorem.}

It will be convenient to write

so that (4.12) is simply

$$
\left.\begin{array}{l}
I_{S} \equiv \int_{S}\left(-n_{i} g^{i}\right) d W_{2} \\
I_{R} \equiv \int_{R}\left(g^{i}\right)_{i} d W_{3},
\end{array}\right\}
$$

$$
I_{S}=I_{R}
$$

Substitute in $I_{R}$ the value of $g^{i}$ given by (2.8). The integrand becomes

$$
\begin{aligned}
\left(g^{i}\right)_{i} & =-\left\{\lambda^{j}\left(\lambda^{i}\right)_{j}\right\}_{i} \\
& =-\left(\lambda^{j}\right)_{i}\left(\lambda^{i}\right)_{j}-\lambda^{j}\left(\lambda^{i}\right)_{j i} \\
& =-\left(\lambda^{j}\right)_{i}\left(\lambda^{i}\right)_{j}-\lambda^{j}\left(\lambda^{i}\right)_{i j}-\lambda^{j} K_{k j i}^{i} \lambda^{k}
\end{aligned}
$$

2 Unless, indeed, $\Delta_{1} f=1$. But in this case the world-lines of the fundamental observers are geodesics, and consequently $g^{i}=0$, so that $(4.10)$ reduces to the trivial identity $0=0$. 
by the permutation-formula

$$
\left(\lambda^{i}\right)_{j k}-\left(\lambda^{i}\right)_{k j}=K_{l j k}^{i} \lambda^{l}
$$

for covariant differentiation ${ }^{1} . \quad K_{l j k}^{i}$ is the Riemann-Christoffel tensor. But $K_{k j i}^{i}=-K_{k j}$, where $K_{k j}$ is the gravitational tensor. So

$$
\left(g^{i}\right)_{i}=-\left(\lambda^{j}\right)_{i}\left(\lambda^{i}\right)_{j}-\left(\lambda^{j}\right)\left(\lambda^{i}\right)_{i j}+K_{k j} \lambda^{k} \lambda^{j}
$$

Hence, if the vector $\lambda^{i}$ is such that

we have

$$
\lambda^{j}\left(\lambda^{i}\right)_{i j}+\left(\lambda^{j}\right)_{i}\left(\lambda^{i}\right)_{j}=0
$$

$$
I_{R}=\int_{R} \lambda^{i} \lambda^{j} K_{i j} d W_{3}
$$

The condition (5.3) imposes a limitation on the nature of the hypersurfaces $f=$ const. which will be considered in $\$ 7$. It may be noticed that the scalar $\lambda^{i} \lambda^{j} K_{i j}$ in the integrand is the mean curvature ${ }^{2}$ of space-time for the direction $\lambda^{i}$; or in other words, it is the sum of the Riemannian curvatures determined by $\lambda^{i}$ and any three mutually orthogonal non-null vectors orthogonal to it.

Now if we neglect the cosmological constant, the field-equations of general relativity are

$$
K_{i j}=-8 \pi \beta\left(T_{i j}-\frac{1}{2} g_{i j} T\right),
$$

where $\beta$ is the Newtonian constant of gravitation, $T_{i j}$ is the energytensor and $T=g^{i j} T_{i j}$. Substituting in (5.4) and using the fact that $\lambda^{i}$ is a unit vector, we get

$$
I_{R}=-8 \pi \beta \int_{R}\left(\lambda^{i} \lambda^{j} T_{i j}-\frac{1}{2} T\right) d W_{3} .
$$

So the generalised Gauss' Theorem may be stated thus:

If $S$ is a closed surface in a spatial section $f\left(x^{i}\right)=$ const. of space. time, and if $R$ is the spatial volume enclosed by $S$, then

$$
\int_{S}\left(-n_{i} g^{i}\right) d W_{2}=-8 \pi \beta \int_{R}\left(\lambda^{i} \lambda^{j} T_{i j}-\frac{1}{2} T\right) d W_{3}
$$

where $-n_{i} g^{i}$ is the outward normal component of gravitational force relative to a set of fundamental observers whose world-lines have the

I Veblen, Invariants of Quadratic Differential Forms (Cambridge Tract No. 24), page 41 (16.1).

2 Eisenhart, Riemannian Geometry (1926), 113. 
direction of the unit vector $\lambda^{i}$, and $d W_{2}, d W_{3}$ are respectively the elements of area and volume relative (in the sense of \$4) to the fundamental observers.

Before proceeding further, it is perhaps desirable to show that (5.7) reduces in the case of the statical space-time

$$
d s^{2}=U d t^{2}-a_{\mu \nu} d x^{\mu} d x^{\nu} \quad\left(\mu, \nu=1,2,3 ; x^{0} \equiv t\right)
$$

to the formula given by Whittaker. In this case

$$
\begin{array}{cl}
g_{00}=U, & g_{\mu \nu}=-a_{\mu \nu}, \quad g_{0 \mu}=0, \\
g^{00}=U^{-1}, & g^{\mu \nu}=-a^{\mu \nu}, g^{0 \mu}=0, \\
-g=a U, &
\end{array}
$$

where $\left(a^{\mu \nu}\right)$ is the matrix reciprocal to $\left(a_{\mu \nu}\right)$, so that $a^{\mu \nu} a_{\nu \sigma}=\delta_{\sigma}^{\mu}$, and $a$ is the determinant $\left|a_{\mu \nu}\right|$. Also the function $f\left(x^{i}\right)$ is simply $f \equiv t$. Consequently

$$
\frac{\partial f}{\partial x^{0}}=1, \quad \frac{\partial f}{\partial x^{\mu}}=0, \quad(\mu=1,2,3)
$$

whence

$$
\Delta_{1} f=g^{00}=U^{-1} \text {. }
$$

Further, by (2.4) $\lambda_{i}=U^{\frac{1}{3}} \partial f / \partial x^{i}$, so by (5.12) we have

$$
\lambda_{0}=U^{\frac{1}{2}}, \quad \lambda_{\mu}=0, \quad(\mu=1,2,3),
$$

and from this and (5.10) we at once deduce that

$$
\lambda^{0}=U^{-\frac{1}{2}}, \quad \lambda^{\mu}=0, \quad(\mu=1,2,3) .
$$

Hence by (2.9) and (3.4) it follows that

$$
\left.\begin{array}{l}
g^{0}=0, n^{0}=0, \\
g_{0}=0, n_{0}=0 .
\end{array}\right\}
$$

The remaining components $g^{\mu}, n^{\mu}$ of these vectors are those of ordinary spatial 3-vectors.

Now the metric of the space $t=$ const. is

$$
d l^{2}=a_{\mu \nu} d x^{\mu} d x^{\nu} .
$$

If the surface $S$ is given parametrically, say by

$$
x^{\mu}=x^{\mu}\left(u^{1}, u^{2}\right), \quad(\mu=1,2,3)
$$

its induced metric is

$$
d l^{2}=a_{\mu \nu} \frac{\partial x^{\mu}}{\partial u^{a}} \frac{\partial x^{\nu}}{\partial u^{b}} d u^{a} d u^{b},
$$

where $a, b$ sum from 1 to 2 only, or

$$
d l^{2}=a_{a b} d u^{a} d u^{b}
$$


where $a_{a b} \equiv a_{\mu \nu} \frac{\partial x^{\mu}}{\partial u^{a}} \frac{d x^{\nu}}{\partial u^{b}}$. We denote the determinant $\left|a_{a b}\right|$ by $a$, and suppose that the rotation from the curves $u^{2}=$ const. to the curves $u^{1}=$ const. is along the outward normal to $S$. Then the unit outward normal-vector is ${ }^{1}$

$$
-n_{\mu}=\frac{1}{2} \epsilon_{\mu \nu \sigma} \epsilon^{a b} \frac{\partial x^{\nu}}{\partial u^{a}} \frac{\partial x^{\sigma}}{\partial u^{b}},
$$

where $\epsilon_{\mu \nu \sigma}= \pm a^{\natural}$ according as $\mu \nu \sigma$ is an even or odd permutation of 123 , and is zero otherwise; and $\epsilon^{a b}= \pm a^{-\frac{1}{b}}$ according as $a b \equiv 12$ or 21 , and is zero if $a$ and $b$ are the same. Also

$$
\left.\begin{array}{l}
d V_{2}=a^{\frac{1}{3}} d u^{1} d u^{2} \\
d V_{3}=a^{\frac{1}{4}} d x^{1} d x^{2} d x^{3}
\end{array}\right\}
$$

Substituting from (5.13), (5.15), (5.16), (5.17) and (5.18) in (5.7), we get on using (4.11) and (5.11),

$$
\begin{aligned}
\int_{S}\left\{g^{1} \frac{\partial\left(x^{2}, x^{3}\right)}{\partial\left(u^{1}, u^{2}\right)}\right. & \left.+g^{2} \frac{\partial\left(x^{3}, x^{1}\right)}{\partial\left(u^{1}, u^{2}\right)}+g^{3} \frac{\partial\left(x^{1}, x^{2}\right)}{\partial\left(u^{1}, u^{2}\right)}\right\}(-g)^{\frac{1}{4}} d u^{1} d u^{2} \\
& =-8 \pi \beta \int_{R}\left(U^{-1} T_{00}-\frac{1}{2} T\right)(-g)^{\frac{1}{2}} d x^{1} d x^{2} d x^{3} \\
& =-8 \pi \beta \int_{R}\left(T_{0}^{0}-\frac{1}{2} T\right)(-g)^{\frac{1}{1}} d x^{1} d x^{2} d x^{3}
\end{aligned}
$$

since $T_{0}^{0}=g^{00} T_{00}=U^{-1} T_{00}$. This is the formula obtained by Whittaker ${ }^{2}$.

§6. Gauss' Theorem for a material field.

Consider now a purely material field of zero pressure. For such a field the energy-tensor is given by

$$
T^{i j}=\sigma_{0} \frac{d \xi^{i}}{d \tau} \frac{d \xi^{j}}{d \tau}
$$

where the $\xi^{i}$ are the coordinates of a particle at its proper-time $\tau$, and $\sigma_{0}$ is the proper-density of matter defined by the invariant condition that

$$
\int \sigma_{0}(-g)^{t} d x^{0} d x^{2} d x^{2} d x^{3}
$$

1 McConnell, Applications of the Absolute Differential Calculus (1931), 197. The negative sign is taken on the left-hand side of (5.17) because $n_{\mu}=g_{\mu \nu} n^{\nu}=-a_{\mu \nu} n^{\nu}$.

${ }^{2}$ Loc. cit., page 388, equation (I). Of. Walker, page 173. 
integrated over any region of space-time, is equal to the sum of the lengths of the world-lines of material particles in that region, each multiplied by the proper-mass of the particle to which it belongs ${ }^{1}$. So

$$
\begin{aligned}
\lambda^{i} \lambda^{j} T_{i j} & =\lambda_{i} \lambda_{j} T^{i j} \\
& =\sigma_{0}\left(\lambda_{i} \frac{d \xi^{i}}{d \tau}\right)^{2} \\
& =\sigma_{0} \cosh ^{2} \theta,
\end{aligned}
$$

where $\theta$ is the angle between the directions in space-time of the world-lines of the particle and local fundamental observer. The hyperbolic cosine is chosen to make $\theta$ real, the directions both being time-like. Also

$$
T=g_{i j} T^{i j}=\sigma_{0},
$$

and (5.7) therefore becomes

$$
\int_{S}\left(-n_{i} g^{i}\right) d W_{2}=-4 \pi \beta \int_{R} \sigma_{0} \cosh 2 \theta d W_{3} .
$$

Consider one of the particles, say that at the world-point $\left(\xi^{i}\right)$. The world-line of one of the fundamental observers passes through $\left(\xi^{i}\right)$ in the direction $\lambda^{i}(\xi)$. Now in a sufficiently small neighbourhood of $\left(\xi^{i}\right)$ space-time is approximately galilean, so, taking $\lambda^{i}$ as the direction of the time-axis in this local galilean space, we quickly deduce from special relativity that

$$
\tanh \theta=v / c,
$$

where, as above, $\theta$ is the angle between the world-lines of the particle and fundamental observer, and $v$ is the instantaneous velocity of the particle relative to the observer; $c$ is the velocity of light, which we are taking to be unity. Substituting in (6.3), we get

$$
\int_{S}\left(-n_{i} g^{i}\right) d W_{2}=-4 \pi \beta \int_{R} \frac{\sigma_{0}\left(1+v^{2}\right)}{1-v^{2}} d W_{3} .
$$

If $v$ is small ${ }^{2}$, the right-hand integral is approximately

$$
\begin{aligned}
I_{R} & =-4 \pi \beta \int_{R} \sigma_{0} d W_{3} \\
& =-4 \pi \beta \int_{R} \sigma_{0} U^{\frac{3}{3}} d V_{3}
\end{aligned}
$$

${ }^{1}$ I am here quoting from Whittaker. Cf. Weyl, Space-Time-Matter (1922), p. 214 (71), or Eddington, Mathematical Theory of Relativity (1924), p. 126.

2 It is not legitimate to put $v$ actually equal to zero. For if it were the world-lines of the fundamental observers would coincide with those of the material particles and would therefore be geodesics, so that ( $c f$. second footnote of $\$ 4$ ) equation (4.10) would reduce to the trivial identity $0=0$ and (5.7) would not in general follow. 
by (4.11) and (2.5), $d V_{3}$ being the true element of 3-volume. Returning to the region $Q$ of space-time considered in $\S 4$, that is, the region bounded by the hypersurfaces $f=t, \phi=0, f=t+d t$, we get, using (4.6),

$$
\begin{aligned}
I_{R} d t & =-4 \pi \beta \int_{Q} \sigma_{0} d \varpi d V_{3} \\
& =-4 \pi \beta \int_{Q} \sigma_{0} d V_{4}
\end{aligned}
$$

by (4.8), $d V_{4}$ being the element of 4 -volume

$$
d V_{4}=(-g)^{\frac{1}{2}} d x^{0} d x^{1} d x^{2} d x^{3} \text {. }
$$

By (6.2) we at once deduce that

$$
I_{R} d t=-4 \pi \beta \sum_{r} M_{r} d \varpi_{r}
$$

where the right-hand side denotes the sum of the proper-masses $M_{r}$ of particles in the 3-dimensional region $R$, each multiplied by the length $d \bar{\sigma}_{r}$ of the world-line of the particle inside the 4-dimensional region $Q$. So

But by (4.6)

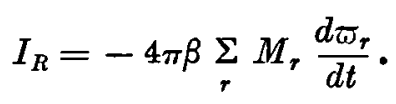

$$
\frac{d \varpi_{r}}{d t}=\left(U_{r}\right)^{\frac{1}{2}}
$$

where $U_{r}$ is the value of the function $U$ at the world-point $\left\langle x_{r}^{i}\right\rangle$ of the particle $M_{r}$ in the region $R$ enclosed by $S$. Substituting in (6.5), we deduce that Gauss' Theorem gives the approximate relation

$$
\int_{S}\left(-n_{i} g^{i}\right) d W_{3}=-4 \pi \beta \sum_{r} M_{r}\left(U_{r}\right)^{\frac{1}{2}}
$$

when the velocity of the particles relative to the fundamental observers is small. The right-hand side denotes $-4 \pi \beta$ times the sum of the proper-masses of the particles inside $S$, each multiplied by the corresponding value of $U^{\frac{1}{2}}$. In the terminology of Whittaker, $M_{r}\left(U_{r}\right)^{\frac{1}{2}}$ is the potential mass of the particle.

Equation (6.6) may be compared with the classical formula (1.1).

\$7. Discussion of the limitation (5.3).

The congruence of curves defined by the vector-field $\lambda^{i}$ was subjected to the limitation

$$
\lambda^{j}\left(\lambda^{i}\right)_{i j}+\left(\lambda^{j}\right)_{i}\left(\lambda^{i}\right)_{j}=0
$$

Since by (2.4) and (2.5) $\lambda_{i}=\frac{\partial f}{\partial x^{i}}\left(\Delta_{1} f\right)^{-\frac{1}{2}}$, this is really a partial 
differential equation which must be satisfied by the hypersurfaces $f=$ const. which define the spatial sections of space-time. In order to investigate the meaning of this limitation, we transform to a new system of coordinates $t \equiv u^{0}, u^{1}, u^{2}, u^{3}$ defined in the following manner. The differential equations of the world-lines of the observers are, by (2.6),

$$
\frac{d x^{i}}{d \bar{\omega}}=\lambda^{i}
$$

Let $\phi_{1}\left(x^{i}\right)=$ const., $\phi_{2}\left(x^{i}\right)=$ const., $\phi_{3}\left(x^{i}\right)=$ const. be three independent solutions of these equations. Then for each choice of the arbitrary constants the three equations $\phi_{\mu}\left(x^{i}\right)=$ const. represent hypersurfaces which intersect in one of the world-lines, and which therefore cut the hypersurfaces $f=$ const. orthogonally.

Make the transformation of coordinates

$$
\begin{aligned}
f\left(x^{i}\right) & =t \equiv u^{0}, \\
\phi_{1}\left(x^{i}\right) & =u^{1}, \\
\phi_{2}\left(x^{i}\right) & =u^{2}, \\
\phi_{3}\left(x^{i}\right) & =u^{3} .
\end{aligned}
$$

Then in the new system of coordinates the metric becomes

with $^{1}$

$$
\begin{aligned}
& d s^{2}=\gamma_{i j} d u^{i} d u^{j} \\
& \gamma_{0 \mu}=0, \quad(\mu=1,2,3) .
\end{aligned}
$$

So (7.3) is of the form

$$
d s^{2}=U d t^{2}-a_{\mu \nu} d u^{\mu} d u^{\nu}
$$

where $U$ and the $a_{\mu \nu}\left(\equiv-\gamma_{\mu \nu}\right)$ are in general functions of $t$ as well as of $u^{1}, u^{2}, u^{3}$, so that (7.5) does not now necessarily represent a statical line-element. The spatial hypersurfaces $f=$ const. are now $t=$ const. and we have as before $(\S 5)$,

$$
\Delta_{1} f=U^{-1}
$$

In the coordinate-system $\left(u^{i}\right)$ the vector $\lambda^{i}$ has components

$$
\lambda^{i} \equiv\left(U^{-\frac{1}{2}}, 0,0,0\right),
$$

(cf. (5.15)). Now by the well-known formula for the contracted covariant derivative of a vector,

$$
\left(\lambda^{i}\right)_{i}=\frac{1}{(-\gamma)^{\frac{1}{2}}} \frac{\partial}{\partial u^{i}}\left[(-\gamma)^{\frac{i}{2}} \lambda^{i}\right]
$$

\footnotetext{
${ }^{1}$ Eisenhart, Riemannian Geometry, p. 43 (14.13).
} 
where $\gamma$ is the determinant $\left|\gamma_{i j}\right|$, that is,

where

$$
\begin{aligned}
-\gamma & =U a \\
a & =\left|a_{\mu \nu}\right| .
\end{aligned}
$$

Using (7.7) we get

$$
\begin{aligned}
\left(\lambda^{i}\right)_{i} & =\frac{1}{(U a)^{\frac{1}{2}}} \frac{\partial a^{\frac{1}{2}}}{\partial t} \\
& =\frac{1}{2 U^{\frac{1}{2}}} \frac{\partial}{\partial t} \log a .
\end{aligned}
$$

So

$$
\begin{aligned}
\lambda^{j}\left(\lambda^{i}\right)_{i j} & =U^{-\frac{1}{2}}\left(\lambda^{i}\right)_{i 0} \text { by (7.7) } \\
& =\frac{1}{U^{\frac{1}{2}}} \frac{\partial}{\partial t}\left(\lambda^{i}\right)_{i}
\end{aligned}
$$

since $\left(\lambda^{i}\right)_{i}$ is a scalar. So by (7.8),

$$
\lambda^{j}\left(\lambda^{i}\right)_{i j}=\frac{1}{2 U^{\frac{1}{2}}} \frac{\partial}{\partial t}\left(\frac{1}{U^{\frac{1}{2}}} \frac{\partial}{\partial t} \log a\right) .
$$

This is the first term of equation (7.1). A similar calculation shows that the second term is

$$
\left(\lambda^{j}\right)_{i}\left(\lambda^{i}\right)_{j}=\frac{1}{4 U} a^{\mu \nu} a^{\sigma \rho} \frac{\partial a_{\mu \sigma}}{\partial t} \frac{\partial a_{\nu \rho}}{\partial t}
$$

where, as usual, $\left(a^{\mu \nu}\right)$ is the matrix reciprocal to $\left(a_{\mu \nu}\right)$. Using the fact that $\frac{\partial}{\partial t} \log a=a^{\mu \nu} \frac{\partial a_{\mu \nu}}{\partial t}$, we therefore deduce that (7.1) is

$$
\frac{1}{2 U^{t}} \frac{\partial}{\partial t}\left(\frac{a^{\mu \nu}}{U^{3}} \frac{\partial a_{\mu \nu}}{\partial t}\right)+\frac{1}{4 U} a^{\mu \nu} a^{\sigma \rho} \frac{\partial a_{\mu \sigma}}{\partial t} \frac{\partial a_{\nu \rho}}{\partial t}
$$

in the coordinate-system $\left(u^{i}\right)$. This equation is obviously satisfied when the space-time (7.5) is statical.

Now the second fundamental form $b_{\mu \nu}$ of the hypersurfaces $t=$ const. is easily shown to have the value ${ }^{1}$

$$
\begin{aligned}
b_{\mu \nu} & =-\frac{1}{2 U^{\frac{1}{2}}} \frac{\partial \gamma_{\mu \nu}}{\partial t} \\
& =\frac{1}{2 U^{\frac{1}{2}}} \frac{\partial a_{\mu \nu}}{\partial t} .
\end{aligned}
$$

So (7.11) becomes

$$
\frac{1}{U^{\frac{1}{2}}} \frac{\partial}{\partial t}\left(a^{\mu \nu} b_{\mu \nu}\right)+a^{\mu \nu} a^{\sigma \rho} b_{\mu \sigma} b_{\nu \rho}=0 .
$$

${ }^{1}$ Eisenhart, op. cit., p. 179, § 2. 
Let now $e_{\mu \nu}$ be the third fundamental form of the hypersurfaces $t=$ const. Then ${ }^{1}$

$$
\begin{aligned}
e_{\mu \nu} & =\gamma^{\sigma \rho} b_{\mu \sigma} b_{\nu \rho} \\
& =-a^{\sigma \rho} b_{\mu \sigma} b_{\nu \rho} ;
\end{aligned}
$$

hence (7.13) becomes

$$
\frac{1}{U^{t}} \frac{\partial}{\partial t}\left(a^{\mu \nu} b_{\mu \nu}\right)-a^{\mu \nu} e_{\mu \nu}=0
$$

If we write $b \equiv a^{\mu \nu} b_{\mu \nu}$ and $e \equiv a^{\mu \nu} e_{\mu \nu}$, then $b$ is the mean curvature of a hypersurface $t=$ const., that is, the sum of its principal normal curvatures $^{2}$. We may call $e$ the third fundamental scalar. Now by (7.7),

$$
\frac{1}{U^{2}} \frac{\partial}{\partial t}=\lambda^{i} \frac{\partial}{\partial u^{i}}
$$

and by (7.2) $\lambda^{i}=\frac{d u^{i}}{\partial \varpi}$ in the coordinate-system $\left(u^{i}\right)$, so

$$
\frac{1}{U^{\frac{1}{2}}} \frac{\partial}{\partial t}=\frac{\partial}{\partial \Phi}
$$

where $d \omega$ is the element of arc of the world-lines of the fundamental observers. So (7.14) gives finally

$$
\frac{\partial b}{\partial \varpi}=e .
$$

This equation is, then, equivalent to (7.1). Evidently the value of $\partial b / \partial \varpi$ at any world-point $P$ is the rate of change, in the direction of the world-line of the fundamental observer passing through $P$, of the mean curvature of the spatial hypersurface which passes through $P$; that is, the rate of change of the mean curvature of "space" with respect to the proper-time of the local observer. Our final conclusion is therefore:

The generalised Gauss' Theorem (5.7) is true only if the spatial hypersurfaces $f=$ const. are so chosen that the rate of change of their mean curvature with respect to the proper-time of the fundamental observers is equal to the third fundamental scalar of the hypersurfaces.

' McConnell, op. cit., p. 202 (29); or Eisenhart, op. cit., p. 219, examples 9 and 10 ; or Levi-Civita, Absolute Differential Calculus (1927), pp. 259, 260.

2 Eisenhart, op. cit., p. 168. 